\title{
Sulfamethazine Sodium
}

National Cancer Institute

\section{Source}

National Cancer Institute. Sulfamethazine Sodium. NCI Thesaurus. Code C84184.

A sodium salt form of sulfamethazine, a sulfonamide antibiotic used in the lifestock industry. 